\title{
NOUVELLE
}

\section{Le récepteur tyrosine kinase c-Kit}

\section{Une nouvelle cible pour les antalgiques?}

Frédéric Marmigère, Frédérique Scamps, Jean Valmier
Inserm U.583, Physiopathologie et thérapie des déficits sensoriels et moteurs, Institut des Neurosciences de Montpellier (INM), Hopital Saint-Eloi, 80, rue Augustin Fliche, BP 74103, 34091 Montpellier Cedex 5, France.

marmigere@montp.inserm.fr
$>$ Le système somato-sensoriel est chargé de la détection des informations émanant de l'environnement interne et externe, et de leur intégration au niveau du système nerveux central. Les neurones sensitifs nociceptifs, ou nocicepteurs, présents dans les ganglions spinaux et trigéminés, sont spécialisés dans la détection de stimulus douloureux thermiques, mécaniques et chimiques, en particulier lors de processus inflammatoires et/ou de traumatismes tissulaires. L'inflammation entraîne l'apparition de plusieurs symptômes douloureux tels que l'allodynie (douleur produite par un stimulus normalement non douloureux) et l'hyperalgésie (douleur exacerbée lors de l'application de stimulus douloureux). En dépit de l'intérêt des douleurs inflammatoires en santé publique, nos connaissances sur les acteurs moléculaires qui confèrent aux nocicepteurs leur identité et leur spécificité fonctionnelle restent encore partielles et les thérapies adaptées font défaut.

\section{Effet pro-algique du NGF et des facteurs de la famille GDNF}

Lors du développement embryonnaire, la survie des neurones nociceptifs dépend du NGF (nerve growth factor) via l'activation de son récepteur à activité tyrosine kinase, TrkA. Chez l'animal, l'invalidation des gènes codant pour le NGF ou TrkA conduit à la disparition de ces neurones [1]. Chez l'homme, des mutations du gène TrkA à l'origine d'une perte de fonction de ce récepteur entraînent une insensibilité congénitale à la douleur [2]. Au cours de la période périnatale, une partie de ces nocicepteurs changent d'identité, cessent d'exprimer les récepteurs TrkA et expriment c-Ret, le récepteur à activité tyrosine kinase des ligands de la famille du GDNF (glial cell line-derived neurotrophic factor): GDNF, artémine, neurturine et perséphine [3].

Chez l'adulte, ces facteurs neurotrophiques régulent également les propriétés fonctionnelles des nocicepteurs et leur expression est augmentée lors de l'inflammation tissulaire. L'injection de NGF, comme celle de GDNF, d'artémine ou de neurturine, induit une hyperalgésie thermique et mécanique, mimant une hyperalgésie inflammatoire [4].

Une des cibles moléculaires de l'effet pro-algique du NGF et de l'artémine est le récepteur TRPVI (transient receptor potential vanilloid 1) [4-6]. En effet, il n'existe pas d'hyperalgésie secondaire à l'inflammation ni d'hyperalgésie thermique induites par le NGF chez les souris dont le gène TRPVI a été invalidé [7-9]. TRPVI est un canal ionique cationique non sélectif activé par des températures supérieures à $43^{\circ} \mathrm{C}$, l'acidité et la capsaïcine, un composé naturel trouvé dans le piment rouge [10-12]. Ce canal est présent sur les terminaisons périphériques de certains nocicepteurs cutanés. Le NGF et les ligands de la famille du GDNF potentialisent l'activité de TRPVl et bloquent sa désensibilisation progressive ou tachyphylaxie, lors d'une activation répétée. Si les mécanismes intracellulaires de régulation de l'expression de TRPVI par le NGF sont encore mal compris et très controversés, des travaux récents indiquent que l'activation de la PI3-kinase et des voies MAP kinases par TrkA entraîne la phosphorylation de TRPVI favorisant l'insertion du canal à la membrane plasmique [13-16].

Implication du récepteur c-Kit dans la douleur inflammatoire

Le récepteur à activité tyrosine kinase c-Kit et son ligand le SCF (stem cell factor) sont exprimés dans une grande variété de tissus où ils modulent une pléthore d'effets biologiques. Cette diversité d'action explique qu'ils aient été proposés comme cible thérapeutique dans plusieurs pathologies, dont I'inflammation allergique. Or une étude récente identifie ce couple comme I'un des modulateurs clé des propriétés fonctionnelles des nocicepteurs, au même titre que les molécules citées précédemment, suggérant son potentiel thérapeutique dans les indications mettant en jeu ces nocicepteurs. II était déjà connu que $10 \%$ des neurones sensitifs exprimant TrkA expriment aussi c-Kit lors de l'embryogenèse, et que le SCF induit la pousse neuritique de ces neurones in vitro [17]. Cependant, la létalité périnatale des souris mutantes pour c-Kit ou le SCF compromet l'analyse des interactions c-Kit/SCF chez l'adulte. Pour contourner ce problème, Milenkovic et al. ont tiré avantage d'une lignée établie de souris mutantes pour c-Kit spontanées, sélectionnées par croisement consanguin intensif entre mutants survivants, et ont croisé ces mutants avec une lignée transgénique surexprimant l'érythropoïétine afin de pallier aux déficits d'hématopoïèse (en particulier l'anémie) causant leur létalité, le taux de survie des souris $\mathrm{C}-\mathrm{Kit}^{-/-}$ passant ainsi de 6 à $26 \%$ [18]. 
Ces auteurs montrent que c-Kit est exprimé par $20 \%$ des neurones des DRG (dorsal root ganglion) adultes parmi lesquels la grande majorité appartient à la population qui exprime TrkA et TRPVl, responsables de la douleur inflammatoire. Bien que le nombre de nocicepteurs et l'innervation cutanée et spinale soient inchangés chez les souris mutantes pour c-Kit, ces animaux montrent une hypoalgésie thermique attribuée à des altérations des propriétés électrophysiologiques d'une sous-population de fibres nociceptives. L'administration chez des souris sauvages d'Imatinib (Glivec ${ }^{\circledR}$ ), un antagoniste de c-Kit, reproduit l'hypoalgésie thermique et les altérations électrophysiologiques observées chez l'animal mutant sans affecter la sensibilité mécanique des nocicepteurs. Enfin, l'administration systémique de SCF chez les souris sauvages et mutantes TRPVI /- provoque une hyperalgésie thermique importante et significative une heure après administration du facteur de croissance, et prolongée pendant 7 heures, uniquement chez l'animal sauvage. Ces résultats in vivo sont confirmés in vitro: des neurones de DRG en culture, mis en présence de SCF, ont une augmentation de l'amplitude du courant induit par la chaleur, une diminution de la température d'activation et une inhibition de la tachyphylaxie induite sur TRPVI par la capsaïcine. Ainsi c-Kit peut être classé parmi le petit nombre de récepteurs à activité tyrosine kinase contrôlant la transduction des stimulus douloureux, groupe auquel appartiennent c-Ret et TrkA. II reste, bien sûr, à déterminer quel est le mécanisme moléculaire à la base de cette régulation et à tester l'effet de l'inhibition pharmacologique de c-Kit sur le comportement induit par une douleur inflammatoire.

\section{En conclusion}

L'ensemble de ces résultats suggère clairement que c-Kit et son ligand SCF sont des acteurs importants dans la transduction normale de la chaleur et l'induction des hyperalgésies thermiques inflammatoires. Puisque des antagonistes de c-Kit sont déjà utilisés dans le traitement de

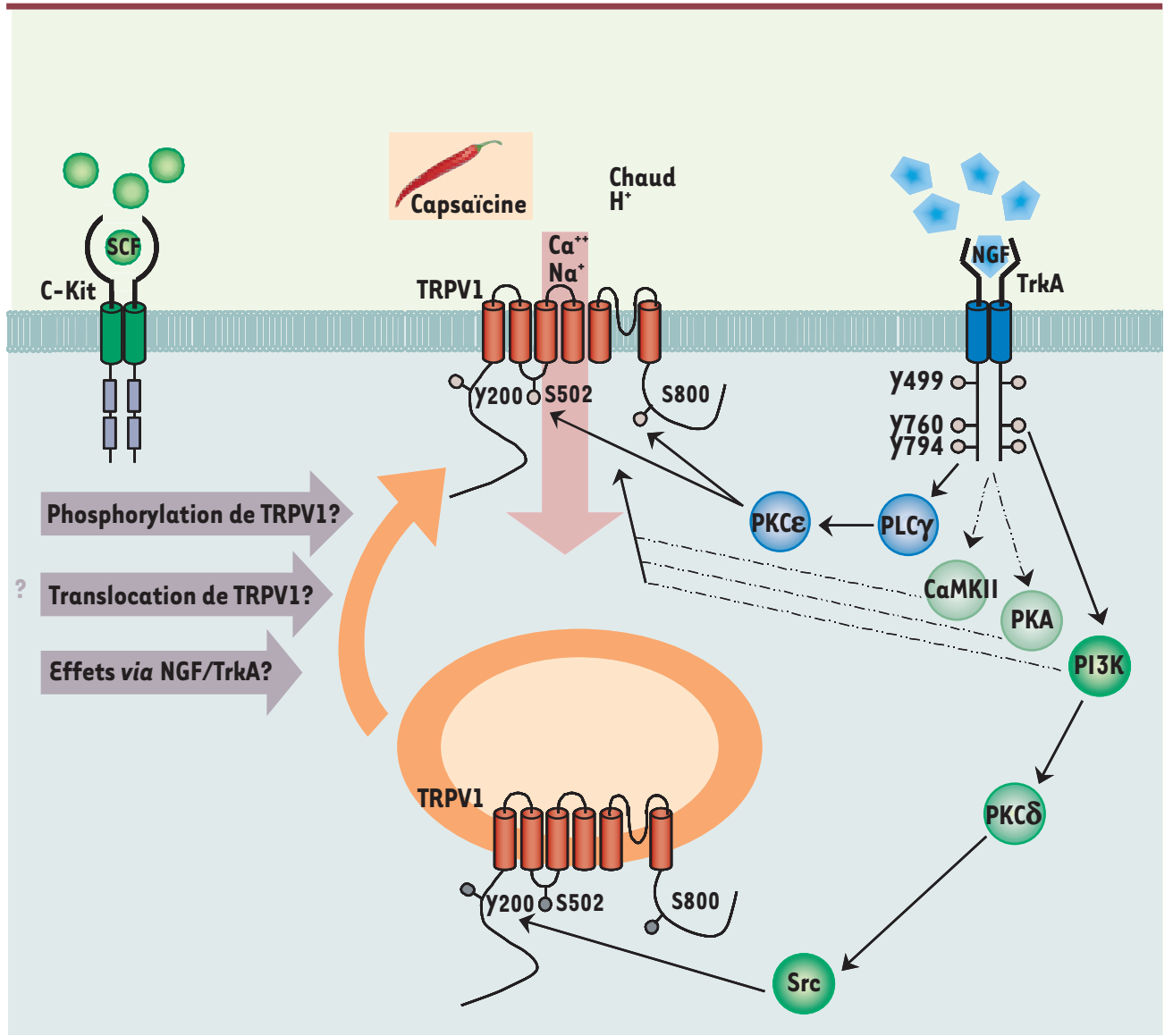

Figure 1. Mécanismes de sensibilisation de TRPVI par le NGF/TrkA et SCF/c-Kit. Dans les terminaisons nociceptives de la peau, le canal ionique TRPVI provoque une entrée de calcium $\left(\mathrm{Ca}^{2+}\right)$ et de sodium $\left(\mathrm{Na}^{+}\right)$lorsqu'il est activé par la chaleur (températures supérieures à $43^{\circ} \mathrm{C}$ ), l'acidité et la capsaïcine. Lors de l'inflammation, l'augmentation locale de NGF potentialise TRPVI par phosphorylation et favorise l'insertion du récepteur à la membrane plasmique via l'activation de TrkA. Les données concernant les voies intracellulaires impliquées dans cette sensibilisation demeurent encore controversées. II a clairement été démontré que l'autophosphorylation de la tyrosine 760 du récepteur TrkA conduit à la phosphorylation de la tyrosine 200 de TRPVI favorisant son insertion à la membrane plasmique par un mécanisme utilisant la phosphoinositide 3-kinase (PI3K), la protéine kinase C delta (PKC $\delta$ ) et Src [14]. Par ailleurs, l'autophosphorylation de TrkA sur la tyrosine 794 active la voie de la protéine kinase $C \gamma$ (PKC $\gamma$ ) et de la phospholipase C epsilon (PLCE) sensibilisant de manière mineure TRPV1 par phosphorylation des résidus sérines 502 et 801 [6, 13, 19]. Enfin, PI3K a aussi été impliquée dans la sensibilisation par NGF/TrkA de TRPVI [15], tout comme la protéine kinase A (PKA) et la calcium calmoduline kinase II (CaMKII) [16, 20] mais ces résultats restent controversés. Enfin, SCF, via l'activation de c-Kit, induit lui aussi une hyperalgésie thermique impliquant TRPVI mais les mécanismes moléculaires de cette activation restent encore à découvrir. 
certaines tumeurs gastrointestinales chez l'homme, la confirmation clinique d'un effet de ces molécules dans le traitement des douleurs inflammatoires devrait nous être fournie dans un futur très proche. $\diamond$ c-Kit tyrosine kinase receptor: a new analgesic target?

\section{RÉFÉRENCES}

1. Snider WD, Wright DE. Neurotrophins cause a new sensation. Neuron $1996 ; 16: 229-32$.

2. Shatzky S, Moses S, Levy J, et al. Congenital insensitivity to pain with anhidrosis (CIPA) in IsraeliBedouins : genetic heterogeneity, novel mutations in the TRKA/NGF receptor gene, clinical findings, and results of nerve conduction studies. Am J Med Genet $2000 ; 92: 353-60$.

3. Molliver DC, Wright DE, Leitner ML, et al. IB4-binding DRG neurons switch from NGF to GDNF dependence in early postnatal life. Neuron 1997 ; 19 : 849-61.

4. Malin SA, Molliver DC, Koerber HR, et al. Glial cell linederived neurotrophic factor family members sensitize nociceptors in vitro and produce thermal hyperalgesia in vivo. J Neurosci $2006 ; 26: 8588-99$.
5. Shu X, Mendell LM. Nerve growth factor acutely sensitizes the response of adult rat sensory neurons to capsaicin. Neurosci Lett $1999 ; 274: 159-62$.

6. Chuang HH, Prescott $\varepsilon D$, Kong $H$, et al. Bradykinin and nerve growth factor release the capsaicin receptor from Ptdlns (4,5)P2-mediated inhibition. Nature $2001 ; 411: 957-62$.

7. Caterina MJ, Schumacher MA, Tominaga M, et al. The capsaicin receptor: a heat-activated ion channel in the pain pathway. Nature $1997 ; 389: 816-24$.

8. Caterina MJ, Leffler A, Malmberg AB, et al. Impaired nociception and pain sensation in mice lacking the capsaicin receptor. Science $2000 ; 288$ : 306-13.

9. Davis JB, Gray J, Gunthorpe MJ, et al. Vanilloid receptor- 1 is essential for inflammatory thermal hyperalgesia. Nature $2000 ; 405$ : 183-7.

10. Szallasi A, Cortright DN, Blum CA, Eid SR. The vanilloid receptor TRPV1 : 10 years from channel cloning to antagonist proof-of-concept. Nat Rev Drug Discov $2007 ; 6: 357-72$

11. Vassort G, Fauconnier J. Les canaux TRP (transient receptor potential) : une nouvelle famille de canaux à expression variée. Med Sci (Paris) 2008 ; 24 : 163-8.

12. Calvino B. L’hypersensibilité à la chaleur au cours de I'inflammation: le rôle du récepteur TRPVI. Med Sci (Paris) $2003 ; 19: 649-51$.

13. Zhuang ZY, Xu H, Clapham DE, Ji RR. Phosphatidylinosito 3 -kinase activates $\varepsilon R K$ in primary sensory neurons and mediates inflammatory heat hyperalgesia through TRPVI sensitization. J Neurosci $2004 ; 24$ : 8300-9.

14. Zhu W, Oxford GS. Phosphoinositide-3-kinase and mitogen activated protein kinase signaling pathways mediate acute NGF sensitization of TRPV1. Mol Cell Neurosci $2007 ; 34: 689-700$.

15. Zhang $X$, Huang J, McNaughton PA. NGF rapidly increases membrane expression of TRPVI heat-gated ion channels. EMBO J 2005 ; 24 : 4211-23.

16. Bonnington JK, McNaughton PA. Signalling pathways involved in the sensitisation of mouse nociceptive neurones by nerve growth factor. J Physiol 2003 $551: 433-46$

17. Hirata T, Morii $\varepsilon$, Morimoto M, et al. Stem cell factor induces outgrowth of c-kit-positive neurites and supports the survival of c-kit-positive neurons in dorsal root ganglia of mouse embryos. Development $1993 ; 119: 49-56$

18. Milenkovic N, Frahm C, Gassmann M, et al. Nociceptive tuning by stem cell factor/c-Kit signaling. Neuron $2007 ; 56: 893-906$

19. Mandadi S, Tominaga T, Numazaki M, et al. Increased sensitivity of desensitized TRPVI by PMA occurs through PKC-epsilon-mediated phosphorylation at S800. Pain 2006; 123 : 106-16.

20. Shu X, Mendell LM. Acute sensitization by NGF of the response of small-diameter sensory neurons to capsaicin. J Neurophysiol 2001; 86 : 2931-8.

\section{Analyser}

Viabilité cellulaire et Apoptose

Gènes rapporteurs

Instrumentation

Mardi 3 juin à 10 Heures

Forum Labo 2008, stand (H18-I19 Hall3), Paris.

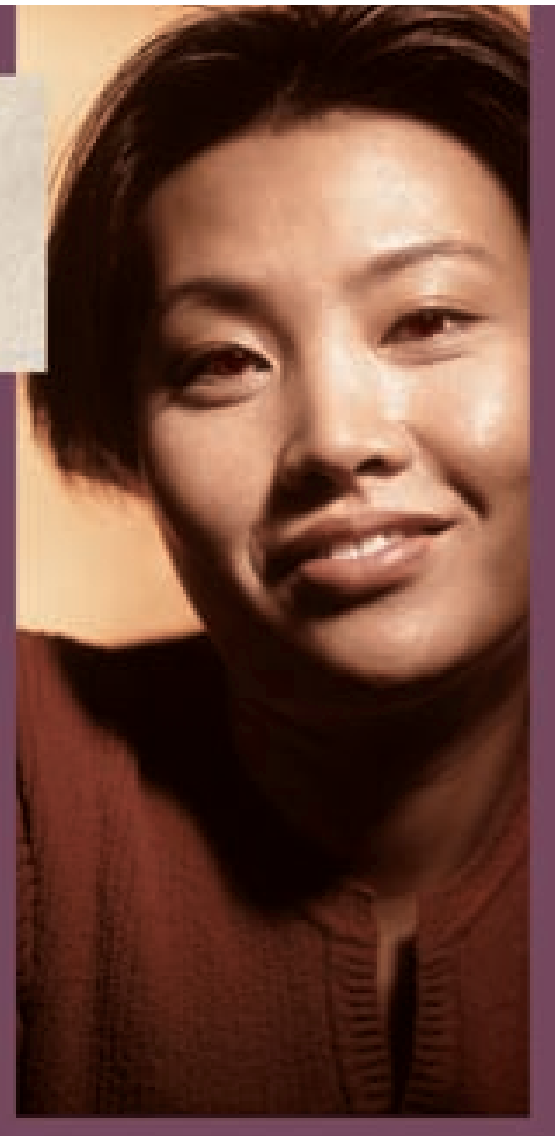

www.promega.com/fr 